\title{
An Interval Type-2 Fuzzy Logic Based System for Improved Instruction within Intelligent E-Learning Platforms
}

\author{
Khalid Almohammadi, Bo Yao, Abdulkareem \\ Alzahrani, Hani Hagras \\ School of Computer Science and Electronic Engineering \\ University of Essex \\ Colchester, UK
}

\author{
Daniyal Alghazzawi \\ Faculty of Computing and Information Technology \\ King Abdulaziz University \\ Jeddah, Saudi Arabia
}

\begin{abstract}
E-learning is becoming increasingly more popular. However, for such platforms (where the students and tutors are geographically separated), it is necessary to estimate the degree of students' engagement with the course contents. Such feedback is highly important and useful for assessing the teaching quality and adjusting the teaching delivery in large-scale online learning platforms. When the number of attendees is large, it is essential to obtain overall engagement feedback, but it is also challenging to do so because of the high levels of uncertainty associated with the environments and students. To handle such uncertainties, we present a type-2 fuzzy logic based system using visual RGB-D features including head pose direction and facial expressions captured from a low-cost but robust 3D camera (Kinect v2) to estimate the engagement degree of the students for both remote and on-site education. This system enriches another self- learning type-2 fuzzy logic system which provides the instructors with suggestions to vary their teaching means to suit the level of course students and improve the course instruction and delivery. This proposed dynamic e-learning environment involves on-site students, distance students, and a teacher who delivers the lecture to all attending onsite and remote students. The rules are learned from the students' behavior and the system is continuously updated to give the teacher the ability to adapt the lecture delivery instructional approach to varied learners' engagement levels. The efficiency of the proposed system has been evaluated through various real-world experiments in the University of Essex iClassroom on a sample of thirty students and six teachers. These experiments demonstrate the efficiency of the proposed interval type-2 fuzzy logic based system to handle the faced uncertainties and produce superior improved average learners' engagements when compared to type-1 fuzzy systems and nonadaptive systems.
\end{abstract}

Keywords-Interval type-2 fuzzy logic systems, elearning student engagement

\section{INTRODUCTION}

The ultimate goal for educational systems is to create an ideal learning environment, which involves good instruction and activities led by the teacher for various numbers of real and distance learners. Unfortunately, there is no single or best instructional approach or tutorial action that teachers can follow to positively affect different learners' engagements within the dynamic e-learning environment. The difficulty of solving this dilemma results from several problems raised by various users and from their interactions with the teachinglearning process. First is the issue of teacher expertise in diagnosing the various users' engagements as well as the best tutorial actions to maintain the varied learners' engagement in a balanced and improved way. Even if the teacher's expertise in promoting learner engagement is high, he/she will have no feedback about the distance learners' engagement. In addition, the total number of students taught makes it difficult for the teacher to evaluate the students' interests and the best instructional approaches to motivate them regarding the learning objective. Similarly, new or beginning teachers step into an unknown world, working under the obligation to teach learners with different needs and levels of engagement, and this variable can cause them apprehension [1].

Starting instructors' movements from pre-service teacher training to professional practice is generally unsettling because there is no steady induction into teaching expertise [1]. New teachers are usually required to teach like experienced teachers and thus face the multiple tasks of being students, instructors, and scientists [2]. Although novices do not have the qualities of experienced teachers, they are expected to meet similar requirements as soon as they enter the field. Sometimes this unfairness goes further, with beginning teachers commonly receiving the most difficult teaching assignments [2]. This creates another challenge for new teachers. Therefore, "beginning teachers leave the field at higher rates than beginning workers in other careers" [3].

Consequently, all of the previously mentioned problems affect learning achievement and student engagement. Recently, with advances in educational technology, adaptive educational systems have attracted considerable interest because of their ability to enhance students' learning performance by delivering instructional content according to the specific student requirements and preferences although these systems are to be used by a single student in asynchronous learning environments [4], [5]. Inspired by this technology which learns what works best for students, we extend a synchronous system to adaptive teaching and training that enables the teachers to learn the behavior of expert teachers in tackling different students' engagement in accordance with some variables of the taught content. This process will open opportunities for professional growth for teachers and enhance instruction, 
which leads to better student achievement and prompts their engagement.

The more the learner interacts and engages with the taught content and the teacher's instructional approach, the more information he or she will gain [6]. Consequently if a course delivery's instructional approaches can gain the students' trust and engagement, the students will definitely learn more [6]. Therefore, automatic and continuous learning within the suited instructional approach as determined by the qualified teacher are essential factors for enhancing and managing varied students' learning engagement and characteristics. Thus, our research seeks to map the best instructional approaches as determined by qualified teachers based on some of the common attributes in lessons and the learners' varied levels of engagement. This learned behavior will be used to train and help the other teachers manage the learning and teaching process to improve instruction.

The effectiveness of any adaptive and intelligent teaching framework depends on the approach used to accumulate information relating to best instructional approaches and also on the way this information is modeled to prepare a smart instruction context [4]. Importantly, the question emerges of how one can ensure precision in evaluating and choosing the appropriate teaching approach to promote and improve the learner engagement. This question is quite critical, due to uncertainties about how accurately teacher decisions about instructional approaches are actually categorized by the learning system as well as the corresponding uncertainties associated with how the resulting instruction is actually decided and administered according to the varied level of learner engagement. In synchronized teaching environments, there are high levels of linguistic uncertainties whereby teachers can interpret and act on the same terms, words, or methods (e.g., lesson difficulty, appropriate teaching style, and approach) in various ways according to the their pupils' varied levels of engagement, knowledge, and expertise in their subject [7]. To tackle the uncertainty that may inhibit the advancement of an efficient learning and teaching context, it is suggested that intelligent e-learning environments should incorporate flexible Artificial Intelligence (AI) methods [7].

To the best of our knowledge, no attempts have been proposed to learn the teaching behavior process according to the varied distance and on-site learners' levels of engagement in the learning environments. Fuzzy logic systems are well known for their ability to generate white box models that can handle high levels of uncertainty. However, the vast majority of fuzzy logic systems employ type-1 fuzzy logic systems, which handle encountered uncertainties based on precise type1 fuzzy sets. In contrast, Interval Type-2 Fuzzy Logic Systems (IT2FLSs) can handle the uncertainties encountered through interval type-2 fuzzy sets, which are characterized by a footprint of uncertainty (FOU) that provides an extra degree of freedom in handling high uncertainty levels.

This paper presents an interval type-2 fuzzy logic-based system that is capable of comprehending various teachers' behavior, which involves their instructional decisions in accordance with various learners' average engagement levels and the difficulty level of taught content in dynamic teaching environments. The type- 2 fuzzy model is first created from data gathered from a number of teaching sessions with different teaching approaches that are conducting by different qualified teachers. The learned type- 2 fuzzy-based model is then used to improve instruction delivery approaches that can be used as supplemental and aid tools to furnish the teaching profession and enhance the learning process. We will show how the proposed system enables the customization of instruction delivery to improve and increase different leaners' engagement and motivation. The proposed system is able to continuously respond and adapt to the various learners' engagement. The efficiency of the proposed system has been evaluated through various real-world experiments in the University of Essex iClassroom with the participation of thirty students and six teachers. These experiments indicate the ability of the proposed type-2 fuzzy logic-based system to handle the faced uncertainties to produce better improved learners' engagements when compared to type-1 based fuzzy systems and non-adaptive systems.

Section II gives a brief overview of the need to consider the teaching approaches used to promote student engagement in the teaching process. A brief overview of interval type-2 fuzzy logic systems is presented in Section III. Section IV explains the proposed type-2 fuzzy logic-based system with learners' engagement feedback for improved instruction within intelligent e-learning platforms. Section V describes the experiments and results, and the conclusions and future work are presented in Section VI.

\section{The neEd to CONSIDER Students' Engagement DegReE ACCORDING TO THE TEACHING APPROACHES IN E-LEANING EENVIRONMENTS}

When studying the appropriate teaching approaches in various teaching settings, there is a need to understand student variables and the manner in which students intend to enhance such variables. A detailed review of learners' personalization variables that require modifications within the learning setting and the principal strategies known as pedagogic personalization employed in managing such variables can be found in [4], [8].

A major pitfall in the modern implementation of e-learning is that the learner models disregard student engagement and do not map delivery needs in terms of suited instructional approach. Gaining the objective feedback from the attendees is the key step in the procedures of education as the interests and capabilities of attendees are different causing high-levels of uncertainties. To model these ambiguities and uncertainties so that personalized education is delivered, various attempts have been done. A conventional non-contact method to estimate the engagement degree is to analyze the eye gaze features. In [9], eye gaze direction is calculated based on $2 \mathrm{D}$ video data using low-cost embedded hardware platform to determine the engagement and reaction of the users in gameplay so that feedbacks can be provided into the gaming user interface and gameplay logic. However, 2D image-based methods are inadequate for returning robust features to complex vision applications such as eye gaze recognition. Therefore, higherlevel systems using multiple hybrid sensors were studied where in [10] [11], wearable sensors embedded into the glasses facing 
towards users' eyes were used to analyze the eye gaze and the interests of the users. However, wearable electronic devices are intrusive and uncomfortable for the users especially those electronic devices are required to deploy near the sensitive parts of the human such as eyes. To address these problems, in this paper, we introduce an engagement estimation system using non-contact and low-cost 3D sensor Kinect v2 which is capable to capture reliable features including head pose direction and hybrid features of face expression enabling the convenient and robust estimation of engagement based on IT2FLS in large-scale online and on-site learning in an unconstrained and naturalistic environment where users are allowed to act freely and move without restrictions.

From the above discussions, it is obvious that incorporating learner engagement as a learner adaption variable enriches the learning environment with a crucial pedagogical dimension. This work presents a cheap and nonintrusive means of measuring varied learners' engagement.

\section{A BRIEF OVERVIEW OF TYPE-2 FUZZY LOGIC SYSTEMS}

The Interval Type-2 Fuzzy Logic System (IT2FLS) depicted in Fig. 1a uses interval type-2 fuzzy sets (such as the type-2 fuzzy set shown in Fig. 1b) to represent the inputs and/or outputs of the FLS. In the interval type-2 fuzzy sets all the third dimension values are equal to one. The use of interval type-2 FLS helps to simplify the computation (as opposed to the general type-2 FLS) [12].

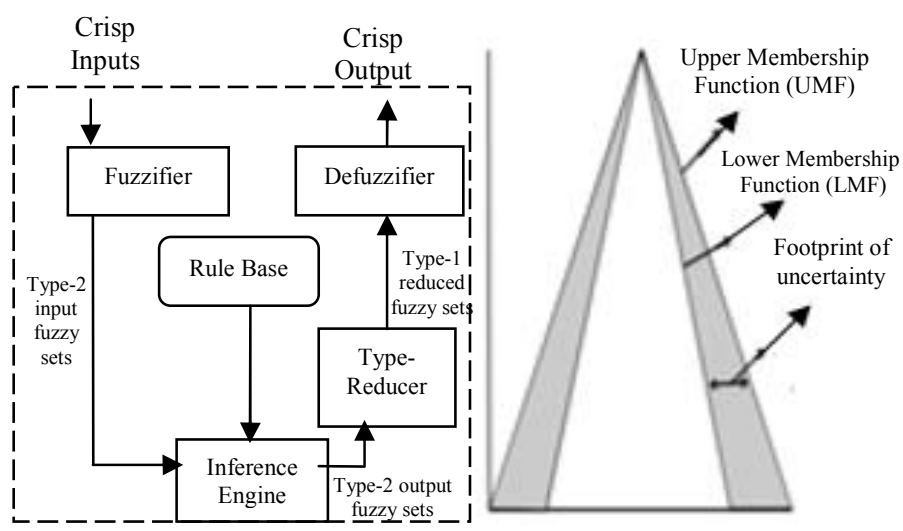

(a)

(b)

Fig. 1. (a) Structure of the type-2 FLS [12] (b) An interval type-2 fuzzy set.

The interval type-2 FLS works as follows: the crisp inputs are first fuzzified into input type-2 fuzzy sets; singleton fuzzification is usually used in interval type-2 FLS applications due to its simplicity and suitability for embedded processors and real-time applications. The input type-2 fuzzy sets then activate the inference engine and the rule base to produce output type- 2 fuzzy sets. The type- 2 FLS rule base remains the same as for the type-1 FLS, but its Membership Functions (MFs) are represented by interval type-2 fuzzy sets instead of type-1 fuzzy sets. The inference engine combines the fired rules and gives a mapping from input type-2 fuzzy sets to output type- 2 fuzzy sets. The type-2 fuzzy output sets of the inference engine are then processed by the type-reducer, which combines the output sets and performs a centroid calculation which leads to type-1 fuzzy sets called the type-reduced sets. There are different types of type-reduction methods. In this paper we will be using the Centre of Sets type-reduction as it has a reasonable computational complexity that lays between the computationally expensive centroid type-reduction and the simple height and modified height type-reductions, which have problems when only one rule fires [12]. After the typereduction process, the type-reduced sets are defuzzified (by taking the average of the type reduced sets) to obtain crisp outputs. More information about the interval type-2 FLS can be found in [12].

In Fig. 1b, the shaded area labelled as Footprint of Uncertainty (FOU) is bounded by lower membership function $\underline{\mu}_{\tilde{A}}(x)$ and an upper membership function $\bar{\mu}_{\tilde{A}}(x)[12]$. Thus an interval type-2 fuzzy set is written as follows:

$$
\tilde{A}=\int_{x \in X}\left[\int_{u \in\left[\underline{\mu}_{\widetilde{A}}(x), \bar{\mu}_{\tilde{A}}(x)\right]} 1 / u\right] / x
$$

\section{THE INTERVAL TYPE-2 BASED SYSTEM WITH LEARNER \\ ENGAGEMENT FEEDBACKS FOR IMPROVED INSTRUCTION WITHIN INTELLIGENT E-LEARNING PLATFORMS}

Across the proposed e-learning environment, knowledge acquisitions would be transformed based on the teacher's instructional approaches and tutorial actions aimed at fulfilling and prompting the current feedback regarding the varied levels of engagement of the distance learners. Fig. 2 demonstrates the conceptual model of the proposed framework whereby the data about the appropriate instructional approach are recorded by the teacher according to the distance learners' varied engagement levels and the lesson's difficulty level (for the three teaching sessions in the case of the conducted experiments) in the observer component. In this component, the data from the e-learning environment are monitored and accumulated at whatever point the instructor changes his or her instructional approach. Accordingly, these collected data will be utilized in the fuzzy learning component. This component will initially enable the system to produce the type-2 fuzzy sets following the methodology demonstrated in [13], [14[, [15]. This method centers on creating type-2 fuzzy sets via the gathering of type-1 fuzzy sets from various teachers. These type-1 fuzzy sets are combined, resulting in the FOU, which appropriately induces a type-2 fuzzy set, which is seen to signify a word. In addition, this component has implemented an unsupervised one-pass approach, as driven by [16], [17], [18], and obtained the rules from the acquired data; this was the main goal of this component. In the adaptation rules component, these learned rules will trigger the best instructional methodologies based on the current state of inputs. This adaptation model component would also consider the new teacher-learned actions that are subject to the existing input parameters from the e-learning environment that are already monitored in the observer component, and will subsequently create an output in consideration of the current state of inputs. This will further enable the online adaptation and enhancement of rules. This facilitates life-long learning owing to the dynamic quality of teaching and learning process interactions. 
As it shown in Fig. 2 there would be 4 components in the proposed system, which would be discussed in the following subsections.

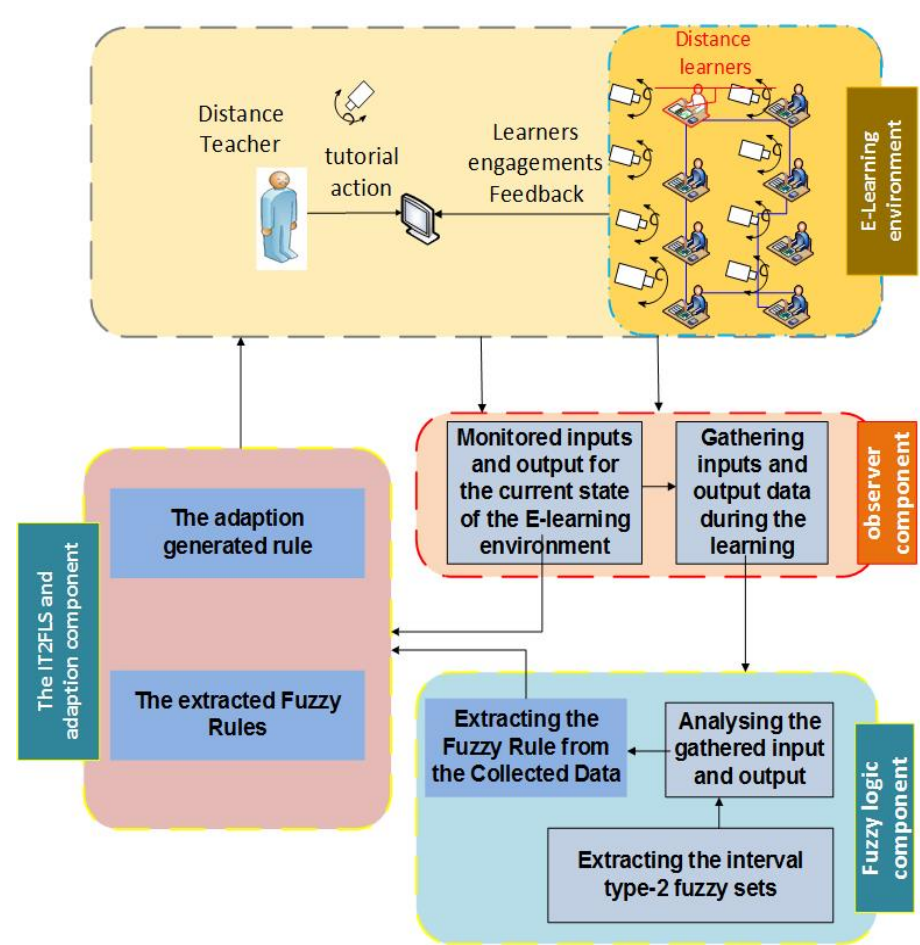

Fig. 2. An overview on the proposed type-2 fuzzy logic based systems with learners' engagement feedbacks for improved instruction within intelligent ELearning platforms.

\section{A. The Observer component}

Primarily, the proposed system gathers and captures the data through collecting the appropriate instructional approach as recorded by the teacher, according to the distance learners' varied average level of engagement and the difficulty level of the current lesson taught within the online learning environment. It is noteworthy that the data (both current inputs and outputs) would be actively recorded by the system if there is any change in the appropriate instructional approach as indicated by the teachers in accordance with the current state of the e-learning environment. Thus, our system creates and learns a descriptive model of the best instructional teachers methodologies that are used in tackling and promoting the varied levels of engagement of distance learners in a balanced way; this is achieved through the data gathered, generating a set of multi-input and multi-output data pairs, which take the following form [16], [17]:

$$
x^{(t)} ; y^{(t)} \quad(t=1,2, \ldots, N) \text {, }
$$

Where $\mathrm{N}$ is referred to as the total of data instances, $x^{(t)} \in R^{n}$, and $y^{(t)} \in R^{k}$. Rules are basically mined by our system, which explains how the $\mathrm{k}$ output, which is the best instructional approach variables, $y=\left(y_{1}, \ldots, y_{k}\right)^{T}$, are affected by the input variables $x=\left(x_{1}, \ldots ., x_{n}\right)^{T}$. A model mapping inputs to outputs is achieved using the established fuzzy rules without requiring a mathematical model. Therefore, individual rules can be adapted online, affecting only certain aspects of the descriptive model created and learned by the proposed system.

1) The proposed method for engagement degree estimation

The first step is to calculate the head pose orientation and the face emotion using the SDK of Kinect v2. After that, the deviation degrees of the current head orientation away from the expected direction (towards the whiteboard or the laptop screen) are calculated to measure the extent of distraction. We then select the largest distraction extent degree to estimate the engagement degree of the student. Finally, based on the deviation and the face emotion, the engagement degree can be computed.

\section{a) Head Pose Estimation}

To robustly estimate the head pose orientation and improve the accuracy of the results, the method based on a regularized maximum likelihood Deformable Model Fitting (DMF) reported in [19] which is robust against the impact of noise factors in the depth channel was employed in this paper. As this method has been developed in the latest version v1409 of Kinect v2 Windows SDK, in our experiments we utilize the module directly to obtain the $3 \mathrm{D}$ head pose orientation of the student in E-Learning environments. In our experiments, we use the latest model Kinect v2 as shown in Fig. 3A which is more robust than the previous model [14]. The SDK of Kinect v2 provides and describes head pose relating to the Kinect camera by three angles: pitch, roll and yaw, as demonstrated in Fig. 3B.

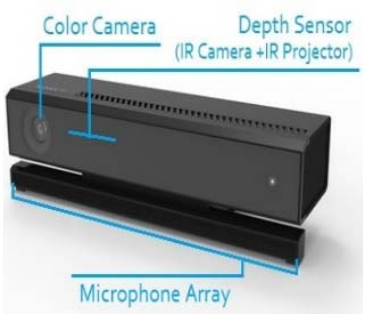

(A)

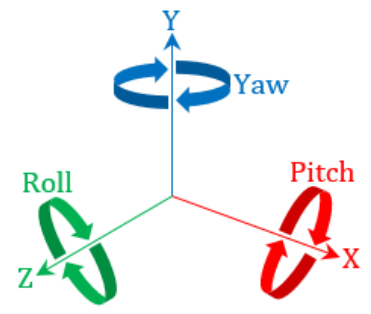

(B) $\begin{array}{lll}\text { Fig. 3. (A) The used Kinect v2. } & \text { (B) Head pose angles }\end{array}$

\section{b) Engagement Degree Estimation}

Based on the visual features including head pose together with the face emotion returned by the $3 \mathrm{D}$ sensor, in our experiments, we will consider the following assumptions describing the relation between the input visual features and the output engagement degree:

- Facing the whiteboard - the student is engaged in the class.

- Facing down - the student is sleepy or probably playing a tablet/smartphone.

- Facing to the left/right - the user is distracted from the learning and interacting with another student nearby.

- Looking around/away - The student is thinking about irrelevant problems and is not concentrated. 
- $\quad$ Face emotion - One eye is not open or both of the two eyes are closed (falling-asleep), and other face emotion for example, mouth open and close (speaking), facial expression is happy, face emotion is engaged, etc.

Based on the assumptions above, the engagement degree of the student can be calculated and modeled by the face emotion of the student and the deviation between the current head orientation and the optimum engaged head pose (facing towards the whiteboard) which are shown in the following equations.

$$
\text { Engagement Degree }=(1-\text { Deviation }) \times \text { Emotion Modifier }
$$

Where Emotion Modifier is decided by the facial emotion including falling-asleep, speaking, happy, engaged. In this experiment we mainly consider the factor falling-asleep for face expression analysis:

$$
\text { Emotion Modifier }\left\{\begin{array}{cc}
1 & \text { Two eyes are open } \\
\text { OEC Modifier } & \text { if } \begin{array}{c}
\text { One eye is closed } \\
0
\end{array} \\
& \text { Two eyes are closed }
\end{array}\right.
$$

Where OEC Modifier is in the range of 0 and 1 , and can be determined by the actual application scenario.

$$
\begin{gathered}
\text { Deviation }=\max \left\{D_{\text {pitch }}, D_{\text {roll }}, D_{\text {yaw }}\right\} \\
D_{\text {pitch }}=\frac{\mid \text { Pitch }_{c}-\text { Pitch }_{o} \mid}{\text { Pitch }_{\text {max }}} \\
D_{\text {roll }}=\frac{\mid \text { Roll }_{c}-\text { Roll }_{o} \mid}{\text { Roll }_{\text {max }}} \\
D_{\text {yaw }}=\frac{\mid \text { Yaw }_{C}-\text { Yaw }_{o} \mid}{\text { Yaw }_{\text {max }}}
\end{gathered}
$$

Where Pitch, Roll $_{c}, Y_{\text {aw }}$ are the three angles (pitch, roll and yaw) of the current head pose obtained by the Kinect v2. Pitch $_{o}$, Roll $_{o}, \mathrm{Yaw}_{o}$ are the angles describing the optimum engaged head pose orientation which are recorded in the training stage. Pitch max $_{\text {mox }}$, Rol $_{\max }, Y_{\text {a }} w_{\max }$ are the maximum angles defined and returned by the Kinect v2 SDK.

\section{B. Fuzzy Learning Rules Component}

Classification of the acquired teaching-learning behavior input/output data through the relevant fuzzy membership functions is a vital step in this component layer. The raw input and output values are ultimately quantified through this process, which leads them into linguistic labels such as low/moderate and high for the average level of engagements. The type-2 fuzzy set extraction approach that is used is indicated in [13], [14], [15], by which a type-2 fuzzy set is developed and its FOU embeds the numerous type-1 fuzzy sets, so that each teacher's individual interpretation can be specified regarding a particular linguistic label that justifies the appropriate instructional approach and engagement level of learners. Therefore, the teachers' diverse views with regard to modeling these words would be integrated by the FOU produced, and the uncertainties would also be handled for the type-2 fuzzy sets. In this method, data are gathered by questioning the teachers regarding their specific linguistic labels through which type-1 fuzzy sets would be produced. Subsequent to this step, the type-2 fuzzy sets are produced, while the type-1 fuzzy sets (demonstrating the teachers' individual views) are integrated, through which the FOU of the type-2 fuzzy set is delivered to represent the given word.

\section{1) Extracting the Fuzzy Rule from the Collected Data}

The data collected from the e-learning environment (input/output) are being combined with the extracted type-2 fuzzy sets so that the rules describing the actions of teachers could be extracted in this section. An enhanced form of the Wang-Mendel technique is used to drive the rule extraction method employed in this paper [16], [17]. This is a one-pass technique for extracting fuzzy rules from the accumulated data. The fuzzy sets for the antecedents and consequents of the rules divide the input and output space into fuzzy regions. Several multi-input/multi-output rules are extracted using the type-2 fuzzy system, through which the association between $x=\left(x_{1}, \ldots ., x_{n}\right)^{T}$ and $y=\left(y_{1}, \ldots ., y_{k}\right)^{T}$ can be explained and take the below form

$$
\text { IF } x_{1} \text { is } \tilde{A}_{1}^{l} \ldots \text { and } x_{n} \text { is } \widetilde{A}_{n}^{l} \text { THEN } y_{1} \text { is } \widetilde{B}_{1}^{l}
$$

$l=1,2, \ldots, M$, Where, $l$ is the index of the rules and $\mathrm{M}$ is the number of rules.

Particularly, for each input $x_{s}$ where $s=1,2, \ldots, n$, there are $V_{i}$ interval type-2 fuzzy sets $\tilde{A}_{s}^{q}, q=1, \ldots, V_{i}$. Moreover, for each output $y_{c}$, there are $V_{o}$ interval type-2 fuzzy sets $\widetilde{B}_{c}^{h}, h=1, \ldots, V_{o}$ where $c=1,2, \ldots, k$.

To clarify and summarize the following representation, an approach comprising a single output is illustrated because of the simplicity of the method for upgrading the rules involving multiple outputs. We mention below, the several stages included in this rule extraction.

Phase 1: The upper and lower membership values are calculated $\bar{\mu}_{\tilde{A}_{s}^{q}}\left(x_{s}^{(t)}\right)$ and $\underline{\mu}_{\tilde{A}_{s}^{q}}\left(x_{s}^{(t)}\right)$ for each of the fuzzy set $\widetilde{A}_{s}^{q}, q=1, \ldots, V_{i}$, and for each input variable $s(s=$ $1, \ldots, n)$ regarding a fixed input-output pair, $\left(x^{(t)} ; y^{(t)}\right)$ in the $\operatorname{dataset}(t=1,2, \ldots, N)$. Find $q^{*} \in\left\{1, \ldots, V_{i}\right\}$ such that $[16$, 17, 14, and 18]:

$$
\mu_{\tilde{A}_{s}^{q^{*}}}^{c g}\left(x_{s}^{(t)}\right) \geq=\mu_{\tilde{A}_{s}^{q}}^{c g}\left(x_{s}^{(t)}\right)
$$

For all $\mathrm{q}=1, \ldots, V_{i}$. Where $\mu_{\tilde{A}_{s}^{q}}^{c g}\left(x_{s}^{(t)}\right)$ is the centre of gravity of the interval membership of $\tilde{A}_{s}^{q}$ at $x_{s}^{(t)}$, which can be seen below [16], [17]:

$$
\mu_{\tilde{A}_{s}^{q}}^{c g}\left(x_{s}^{(t)}\right)=\frac{1}{2}\left[\bar{\mu}_{\tilde{A}_{s}^{q}}\left(x_{s}^{(t)}\right)+\underline{\mu}_{\tilde{A}_{s}^{q}}\left(x_{s}^{(t)}\right)\right]
$$

The given below rule will be known as the rule generated by $\left(x^{(t)} ; y^{(t)}\right)[16],[17]$ :

$$
\text { IF } x_{1} \text { is } \tilde{A}_{1}^{q^{*}(t)} \text {. and } x_{n} \text { is } \tilde{A}_{n}^{q^{*}(t)} \text { THEN } y \text { is centered at } y^{(t)}
$$


For all of the input variables $x_{s}$, there are $V_{i}$ type-2 fuzzy sets $\tilde{A}_{s}^{q}$, which makes the greater amount of possible rules equal to $V_{i}^{n}$. However, when considering the dataset, there will be the generation of those rules amongst the $V_{i}^{n}$ possibilities that show a dominant region comprising a minimum of one data point.

In the first phase, there is the generation of one rule for each particular input/output data pair, with the fuzzy set selected being that which is seen to obtain the greatest value of membership at the data point, and particularly selected as the one in the rule's IF element. But this is not the final version of the rule, which will be computed in the following step. The calculation of the rule weight is accomplished as follows [16], [17]:

$$
w i^{(t)}=\prod_{s=1}^{n} \mu_{\tilde{A}_{s}^{q}}^{c g}\left(x_{s}^{(t)}\right)
$$

A rule $w i^{(t)}$ weight is a degree of the strength of the points $x^{(t)}$ regarding the fuzzy region covered by the entire rule.

Phase 2: For all of the data points from 1 to $N$, the first phase is repeated. With the help of this practice, $N$ rules extracted from the data are taken in the form of Equation (12). Phase 1 witnesses the generation of multiple rules, all of which have the same IF part in common but which are all conflicting. During this phase, those rules that have the same IF part are amalgamated to form a single rule. Subsequently, the rules $N$ are divided into groups, with rules in each of the groups seen to have the same IF part. If it is considered that such groups amount to $M$, and it may also be stated that the group has $N_{l}$ rules, therefore [14], [16], [17], [18]:

$$
\text { IF } x_{1} \text { is } \widetilde{A}_{1}^{l} \ldots \text { and } x_{n} \text { is } \widetilde{A}_{n}^{l} \text { THEN } y \text { is centered at } y^{\left(t_{u}^{l}\right)}
$$

Where $u=1, \ldots, N$ and $t_{u}^{l}$ are the data points index of Group $l$. The equation given below shows how to calculate the weighted average of all rules involved in the conflict group:

$$
a v^{(l)}=\frac{\sum_{u=1}^{N_{l}} y^{(}\left(t_{u}^{l}\right)_{w i}\left(t_{u}^{l}\right)}{\sum_{u=1}^{N_{l}} w i\left(t_{u}^{l}\right)}
$$

Subsequently, a single rule is formed by integrating these $N_{l}$ rules, resulting in the following form [16, 17, 14, 18]:

$$
\text { IF } x_{1} \text { is } \widetilde{A}_{1}^{l} \ldots \text { and } x_{n} \text { is } \widetilde{A}_{n}^{l} \text { THEN } y \text { is } \widetilde{B^{l}}
$$

Where there is the selection of the output fuzzy set $\widetilde{B^{l}}$ on the basis of the following: amongst the $V_{o}$ output interval type2 fuzzy sets $\widetilde{B^{l}}, \ldots, \widetilde{B^{V_{o}}}$, calculate the $B^{h *}$ such that [16], [17]:

$$
\begin{array}{r}
\mu_{B^{h *}}^{c g}\left(a v^{(l)}\right) \geq \mu \frac{c g}{B^{h}}\left(a v^{(l)}\right) \\
\text { for } h=1,2 \ldots, V_{o}
\end{array}
$$

$\widetilde{B^{l}}$ is chosen due to the $B^{h *}$, where $\mu_{B^{h}}^{c g}$ is the centre of gravity of the interval membership of $\widetilde{B^{h}}$ at $a v^{(l)}$ as illustrated in Equation (11).

The proposed system can effectively handle the data pairs of input/output, including multiple outputs as per the work presented above. Stage 1 is recognized as being distinct with regard to the number of outputs associated with each rule. By contrast, Stage 2 provides a straightforward expansion with the aim of enabling rules to encompass multiple outputs; for each output, the calculations detailed in Equations (15), (16), and (17) are repeated.

\section{The IT2FLS and adaption component}

The generated type-2 fuzzy sets and the fuzzy rules extracted from the input and output gathered data of learners enables the proposed system to learn and obtain the best instructional approaches in accordance to the varied level of engagement of the learners and the difficulty level of the taught content. As a result, the system is afterwards in a position to notify the teachers to re-adjust the online learning environment with specific consideration to appropriate instructional approach. The system actions are triggered through the examination and monitoring of various learners varied level of engagements and the lesson difficulty and, subsequently impacts the online instructional environment, with a particular consideration to the learned approximation of best tutorial actions that could be followed by the teachers. Given below are the functionalities of the proposed type- 2 fuzzy adaptive system:

- As specified in the e-learning environment, the crisp inputs including the learners' variables are fuzzified (via singleton fuzzification) into the input interval type2 fuzzy sets.

- The outputs (instructional approaches) type-2 fuzzy sets are generated by the activation of inference engine and rule base.

The proposed system must have the ability to fine-tune with respect to the dynamic and diverse varied learners engagements and various difficulties of the taught lessons states by continuously enabling teachers to modify their instructional approaches. Subsequently, the system will re-adjust its procedures or it would apply new ones. If no rules arouse from the rule base, (i.e. the rule's firing strength in Equation $\left.(13) w i^{(t)}=0\right)$ in a given input, subsequently the system input would be captured by the system and for creating a rule covering this uncovered input status, it will capture the appropriated teaching approaches. Therefore, new rules would be integrated in the system while there is an undefined state of the online learning environment at that moment as per the existing rules in the rules base (i.e. where none of the present rules are fired). The new rules will be generated and the system integrates them in such an instance, in which the online learning environment's current input states are specified by the antecedents besides the consequent fuzzy sets that are dependent on the current state of the instructional approach. The fuzzy sets that have membership values, where $\mu_{\tilde{A}_{C}^{h}}^{c g}\left(x_{s}^{\left(t^{\prime}\right)}\right)>0$ are identified for all of the input parameters $x_{s}$. 
Consequently, for each input parameter, numerous identified fuzzy set(s), are generated in the form of a grid. From such a grid, there is the generation of new rules based on all individual combinations of successive input fuzzy sets. The consequent fuzzy set which provide the greatest value of membership to the teacher defined appropriated instructional approach $\left(y_{c}\right)$ so that it can operate as the generated rule consequent. After performing a calculation of the output interval memberships' center of gravity, we can establish the fuzzy sets [16], [17].

$$
\mu_{\tilde{B}_{c}^{h^{*}}}^{c g}\left(y_{c}\right) \geq \mu_{\tilde{B}_{c}^{h}}^{c g}\left(y_{c}\right)
$$

For $h=1, \ldots, W$ the $\tilde{B}_{c}$ is chosen as $\tilde{B}_{c}^{h^{*}}$ where $c=$ $1, \ldots, k$. Consequently, new and upcoming rules can be progressively added.

In case if the teacher needs to change the suited instructional approaches at a given input status, the fired rules will be identified and the rule consequents will be changed (if more than two teachers signal the same modifications for the teaching approaches) as indicated by Equation (18). Therefore, the fired rules are modified so that the updated suited instruction approaches for the students could be reflected in a desirable way, while taking into account the existing state of the online learning environment. The system proposed in this paper will adopt life-long learning through facilitating the adaptation of rules according to the optimized instruction delivery approaches by teachers, which notably change over time based on the student varied level of engagements, and in regard to the state of the online learning environment. Owing to the system flexibility, the fuzzy logic model learned initially may be effortlessly expanded in order to make changes to both new and existing rules. These fuzzy rules enable a large range of values for all parameters (input and output) to be captured, which in turn enables the continuation of the generation of rules, even when the online learning environment gradually changes. On the other hand, if notable changes occur in terms of the students varied average level of engagements or in the environment (which may not be captured by the present rules, as highlighted above), the new rules will be automatically generated, which ultimately satisfy present conditions. Accordingly, the inconspicuous system will expand its actions and may be adapted in order to improve the instruction delivery.

\section{EXPERIMENT AND RESULTS}

We have performed various real world experiments in the intelligent Classroom (iClassroom) at the University of Essex. The experiments aimed to compare the proposed interval type2 fuzzy logic-based system with the type-1 fuzzy logic-based counterpart system and the non-adaptive version of the system in terms of optimizing the instruction, which leads to better student engagement and satisfaction. The experiments involved choosing 20 lessons from an MS Excel course that were classified in five different difficulty levels (i.e., very hard, hard, moderate, easy, and very easy). In addition, we examined four teaching approaches, namely teaching using PowerPoint slides, practical explanation (demo), teaching with cases (problem solving), and asking questions. These four teaching approaches have been suggested by different expert teachers to be used in the system.

Real-world experiments have been carried out with a sample of 30 students and six teachers from the University of Essex. The experiments started by training the system, we divided a number of 15 students randomly into three groups. Each group had 5 distance learners. Importantly, each group was taught by a different expert teacher instructing a variety of 20 lessons using the four teaching approaches. During the teaching sessions, the learners' average levels of engagement and the average standard deviation level were accumulated every 5 seconds, as well as the difficulty level of the current lesson being presented in the teacher user interface to be used as input variables. When the teacher decided to change the teaching approach, he/she ranked and prioritized these teaching approaches from zero (not beneficial in the current situation) to ten (absolutely beneficial in the current situation) and that ranking was used as an output. The teacher recorded the inputs and their related output in the system's database. These inputs/outputs were captured by the observer component whenever the teacher changed or recorded the appropriate instructional approach. The left hand side of Fig. 4 shows the teachers teaching the lessons, while the right hand side of Fig. 4 shows the students' engagement degree shown in the teacher user interface. The average engagement degree for each student was measured using the Kinect camera (as shown in Fig. 4 and as explained in section IV).

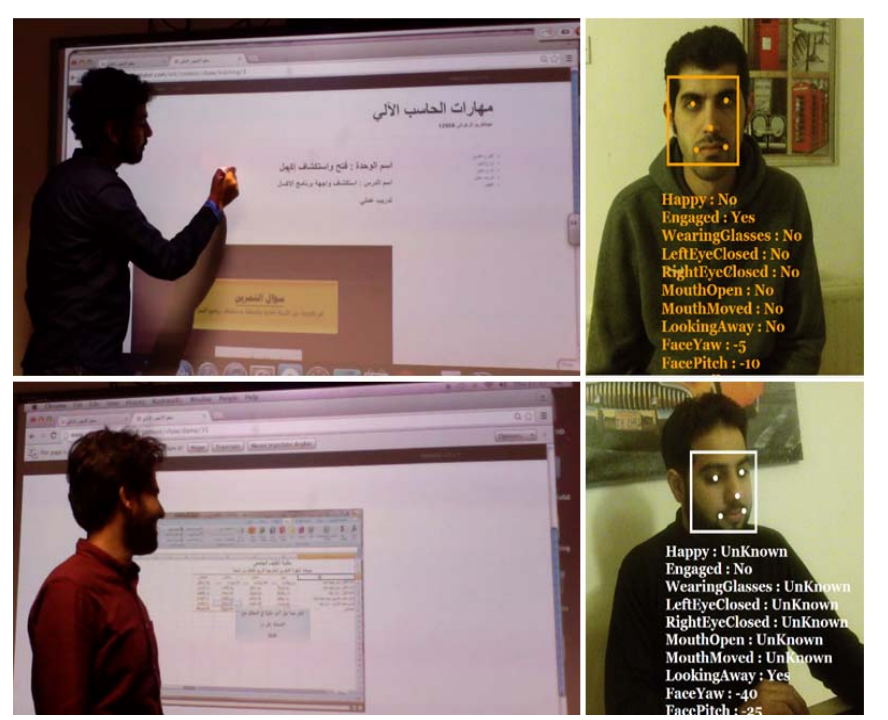

Fig. 4. Teachers are shown on the left side photographs while they are teaching different lessons with different teaching approaches. On the right side photographs, the students' engagement feedback are shown in the teachers' user interface.

It should be noted that the calculation of the average learners' engagement and the standard deviation were taken from the beginning of a lesson being taught in one of the four teaching approaches until another lesson is taught with a different difficulty level or with a change in the teaching approach.

After collecting sufficient datasets, we started the testing phase. Here, three 5-member groups were taught by three 
different teachers. The teacher in the first group used system applied type-1 fuzzy logic while the second group's teacher used the applied interval type- 2 system. The third group was the control group, which was taught with no use of intelligent technology. After dividing the three groups equally, the input and output data for type- 1 and type- 2 groups were obtained. Then, by using the linguistic variables and rules, the fuzzy logic models for both the type-1 and type-2 groups were constructed. The type- 2 fuzzy sets (shown with the thick line in Fig. 5) were obtained to capture the uncertainty that signifies teachers' views concerning a particular linguistic label explaining the average of students' engagement, their standard deviation, and the teaching approach, while the type-1 fuzzy logic system uses a type-1 fuzzy set (shown with the dashed lines) as shown in Fig. 5.

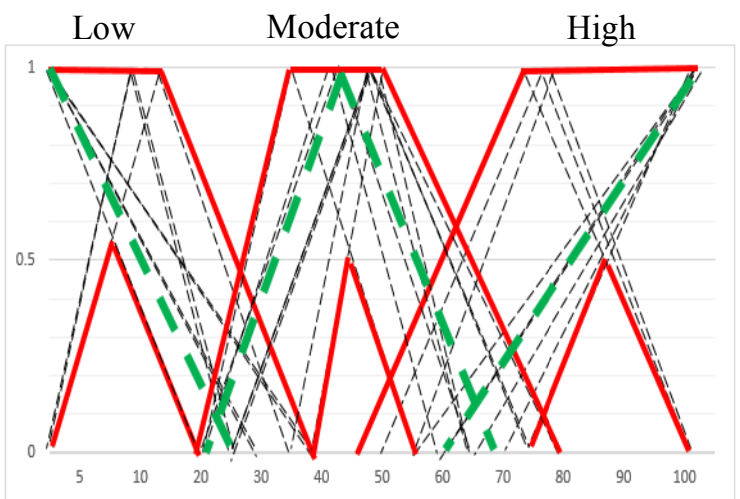

Fig. 5. The generated interval type- 2 fuzzy sets of the average engagment level (think solid lines) and the type-1 fuzzy sets (thick dashed lines).

As soon as the teachers in the first and second groups started introducing the first lesson, the observer component started calculating the average engagement and the standard deviation. At the same time, the observer component tried finding the matched rule(s) with the current monitored inputs. When the system found the matched rule(s), it was presented in the teacher user interface, thus he/she knew what the best teaching approach was in the situation. The teacher could ignore that rule and the system would learn from his/her decision of re-prioritizing and re-ranking the teaching approaches based on the current given data. Hence, if the teacher determined to continue teaching the lesson (or any lesson at the same difficulty level) without changing the teaching approach, the observer component continues calculating the average engagement and the standard deviation. In contrast, when the teacher changed the teaching approach or taught a lesson that differed from the previous one, the observer component would start from zero.

Finally, for evaluation purposes, the teacher's learned data was collected to be compared to the type- 2 and type- 1 fuzzy logic systems to know the average error and standard deviation of the teacher preferred output and the system outputs. In addition, the comparison between the three groups in terms of the average engagements and standard deviations involved comparing them based on the data gathered by the observer component (during the whole teaching session for every group) and based on the students' views that were tracked by their questioner's responses.
TABLE I. AVERAGE ERROR AND STANDARD DEVIATION OF SOME OF THE SYSTEM OUTPUTS

\begin{tabular}{|l|l|l|l|c|}
\hline \multirow{2}{*}{ Output Name } & \multicolumn{2}{|c|}{ Type-2 Fuzzy Logic } & \multicolumn{2}{c|}{ Type-1 Fuzzy Logic } \\
\cline { 2 - 5 } & $\begin{array}{l}\text { Average } \\
\text { error }\end{array}$ & $\begin{array}{c}\text { Standard } \\
\text { deviation }\end{array}$ & $\begin{array}{c}\text { Average } \\
\text { error }\end{array}$ & $\begin{array}{c}\text { Standard } \\
\text { deviation }\end{array}$ \\
\hline $\begin{array}{l}\text { practical } \\
\text { explanation } \\
\text { (demo) }\end{array}$ & 1.90 & 1.28 & 2.78 & 1.67 \\
\hline $\begin{array}{l}\text { teaching with } \\
\text { cases } \\
\text { (problem } \\
\text { solving) }\end{array}$ & 2.09 & 1.32 & 2.88 & 1.79 \\
\hline
\end{tabular}

First, based on the teachers' learned data, TABLE I. shows the average error and standard deviation to compare the teacher preferred output and the system outputs in both type- 2 and type-1 fuzzy logic systems (on account of the space limitations, we present only a sample of the output). These results clearly show that the type-2 fuzzy logic system has less of an average error and standard deviation. Consequently, the type-2 fuzzy logic system appears to be more effective than the type-1 fuzzy logic system in recording teachers' tutorial actions.

On the other hand, according to data gathered by the observer component, the results approve that the use of interval type-2 fuzzy logic makes the students more engaged (68.75 average engagement degree) than the use of type-1 fuzzy logic (64.23 average engagement degree) and the control group (44.34 average engagement degree). Furthermore, the use of the standard deviation of the data reveals that interval type-2 fuzzy logic makes students close to each other in terms of their engagement as shown in Fig. 6, which means that there was not much dispersion of the set of type-2 group's engagement data value. However, the control and type-1 FLS groups' engagements have a notable variation.

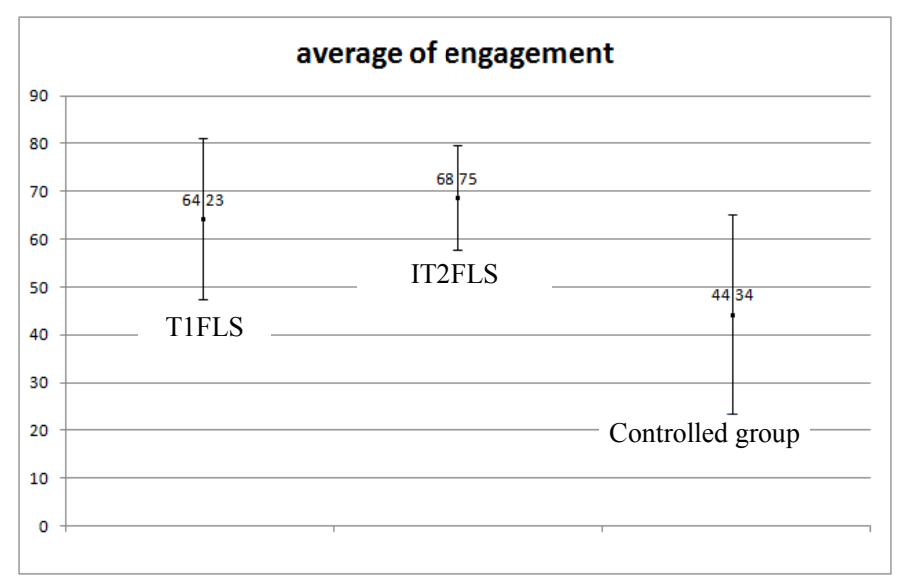

Fig. 6. The average of engagement and standard deviation of the type-1, type-2 Fuzzy logic, and control group based on the data gathered by the observer component using the cameras.

Furthermore, we analyzed the participants' satisfaction and engagement in the questioner using Anova to compare the responses from the groups at a significance level of 0.05 . The analysis revealed that there is a significant difference between the various groups $(\mathrm{p}<<0.05)$. We also carried out multiple 
comparison tests (Tukey and LST) to see which pair of groups has a difference. We observed that group 2 (interval type-2) and 3 (control group) were the most significantly different groups as compared to other pairs and as shown in Fig. 7.

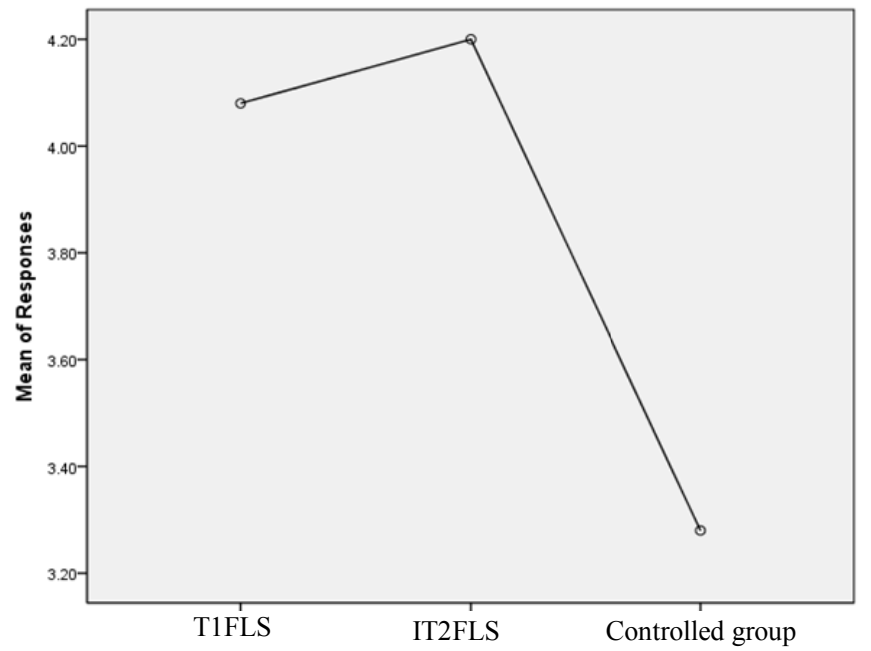

Fig. 7. plot for group means comparison.

\section{CONCLUSIONS AND FUTURE WORK}

In this paper, we presented an interval type-2 fuzzy logic based system, which has the ability of learning different teacher's pedagogical decisions based on the content difficulty level as well as the students' average level of engagement and the variation between the engagement in a dynamic, real online teaching environment. This learned type- 2 fuzzy-based model was applied to enhance the teaching performance by informing the teacher about the best teaching approaches in order to gain an enhanced average level of learners' engagement. Moreover, we presented a method based on type- 2 fuzzy logic utilizing visual RGB-D features including head pose direction and face expressions captured from a low-cost, but robust, 3D camera (Kinect v2) to estimate the engagement degree of the students in both remote and on-site locations. The system has been evaluated and compared with the type-1 fuzzy system and the non-adaptive system. Various real world experiments have been conducted with the participation of 6 teachers and 30 students. The results revealed that the use of the type-2 fuzzy logic system better handled the uncertainties faced in facilitating instruction delivery to the learners, thus helping to promote and increase the average level of engagement more than the type-1 fuzzy systems and the non-adaptive version.

For our future work, we aim to employ general type-2 fuzzy logic to be able to better handle and model the various faced uncertainties. We aim also to deploy the proposed system for eLearning courses including thousands of students.

\section{REFERENCES}

[1] K. Smith and O. Sela, "Action research as a bridge between preservice teacher education and in-service professional development for students and teacher educators," Eur. J. Teach. Educ., vol. 28, no. 3, pp. 293-310, Oct. 2005.
[2] M. ÖZTÜRK and A. Yildirim, "Adaptation Challenges of Novice Teachers," Hacet. Üniversitesi Eğitim Fakültesi Derg., vol. 28, no. 28-1, 2013.

[3] M. E. Wonacott, "Teacher Induction Programs for Beginning CTE Teachers. In Brief: Fast Facts for Policy and Practice," National Dissemination Center for Career and Technical Education, Columbus, OH., Jan. 2002.

[4] V. J. Shute and D. Zapata-Rivera, "Adaptive educational systems," Adaptive technologies for training and education, pp. 7-27, 2012.

[5] White paper based upon the Speak Up 2011 national findings, "Leveraging Intelligent Adaptive Learning to Personalize Education," Intelligent Adaptive Learning : Speak Up Reports, 2012.

[6] R.C. Clark and R.E. Mayer, E-Learning and the Science of Instruction: Proven Guidelines for Consumers and Designers of Multimedia Learning, 3rd ed., San Francisco, USA: John Wiley \& Sons, 2011.

[7] A. Ahmad, O. Basir, and K. Hassanein, "Adaptive user interfaces for intelligent e-Learning: issues and trends," Proceedings of the Fourth International Conference on Electronic Business, 2004, pp. 925-934.

[8] F. Essalmi, L. J. B. Ayed, M. Jemni, and S. Graf, "A fully personalization strategy of E-learning scenarios," Computers in Human Behavior, Elsevier, vol. 26, no. 4, pp. 581-591, 2010.

[9] P. M. Corcoran, F. Nanu, S. Petrescu, and P. Bigioi, "Real-time eye gaze tracking for gaming design and consumer electronics systems," IEEE Transactions on Consumer Electronics, vol. 58, no. 2, pp. 347-355, 2012.

[10] A. Mayberry, P. Hu, B. Marlin, C. Salthouse, and D. Ganesan, "iShadow: design of a wearable, real-time mobile gaze tracker," Proceedings of $12^{\text {th }}$ annual international conference on Mobile systems, applications, and services, 2014, pp. 82-94.

[11] Z. Ye, Y. Li, A. Fathi, Y. Han, A. Rozga, G.D. Abowd, and J. M. Rehg, "Detecting eye contact using wearable eye-tracking glasses," Proceedings of the ACM Conference on Ubiquitous Computing, 2012, pp. 699-704.

[12] J. Mendel, Uncertain Rule-Based Fuzzy Logic Systems: Introduction and New Directions, Prentice Hall PTR, Prentice Hall Inc, 2001.

[13] F. Liu, and J. Mendel, "An interval approach to fuzzistics for interval type-2 fuzzy sets," Proceedings of the 2007 IEEE International Conference on Fuzzy Systems, 2007, pp. 1030-1035.

[14] K. Almohammadi, B. Yao, and H. Hagras, "An interval type-2 fuzzy logic based system with user engagement feedback for customized knowledge delivery within intelligent E-learning platforms," Proceedings of the 2014 IEEE International Conference on Fuzzy Systems, 2014, pp. 808-817.

[15] K. Almohammadi and H. Hagras, "An Interval Type-2 Fuzzy Logic Based System for Customised Knowledge Delivery within Pervasive ELearning Platforms," Proceeings of the 2013 IEEE International Conference on Systems, Man, and Cybernetics, 2013, pp. 2872-2879.

[16] L. X. Wang, "The MW method completed: A flexible system approachto data mining," IEEE Transactions on Fuzzy Systems, vol. 11, no. 6, pp. 768-782, December 2003.

[17] H. Hagras, F. Doctor, A. Lopez and V.Callaghan, "An incremental adaptive life long learning approach for type-2 fuzzy embedded agents in ambient intelligent environments," IEEE Transactions on Fuzzy Systems, vol. 15, no. 1, pp. 41-55, February 2007.

[18] K. Almohammadi and H. Hagras, "An adaptive fuzzy logic based system for improved knowledge delivery within intelligent E-Learning platforms," Proccedings of the the 2013 IEEE International Conference on Fuzzy Systems, 2013, pp. 1-8.

[19] Cai, Qin, David Gallup, Cha Zhang, and Zhengyou Zhang, "3d deformable face tracking with a commodity depth camera." In Computer Vision-ECCV 2010, pp. 229-242. Springer Berlin Heidelberg, 2010. 